\title{
Les relations roumano-grecques dans l'entre-deux-guerres : quelques considérations sur un rapprochement continu
}

The Romanian-Greek Relations during the Interwar Decades: A few

considerations regarding a continuous rapprochement

RelaŢiile româno-elene în perioada interbelică. Câteva consideraŢii privind o

apropiere continuă

\section{Radu Tudorancea}

\section{OpenEdition}

Journals

Édition électronique

URL : https://journals.openedition.org/ceb/9705

DOI : $10.4000 /$ ceb.9705

ISSN : 2261-4184

Éditeur

INALCO

Édition imprimée

ISBN : 978-2-85831-239-9

ISSN : 0290-7402

Référence électronique

Radu Tudorancea, «Les relations roumano-grecques dans l'entre-deux-guerres : quelques considérations sur un rapprochement continu », Cahiers balkaniques [En ligne], 44 | 2016, mis en ligne le 28 novembre 2017, consulté le 07 juillet 2021. URL : http://journals.openedition.org/ceb/9705 ;

DOI : https://doi.org/10.4000/ceb.9705

Ce document a été généré automatiquement le 7 juillet 2021

Cahiers balkaniques est mis à disposition selon les termes de la Licence Creative Commons Attribution - Pas d'Utilisation Commerciale 4.0 International. 


\title{
Les relations roumano-grecques dans l'entre-deux-guerres : quelques considérations sur un rapprochement continu
}

\author{
The Romanian-Greek Relations during the Interwar Decades: A few \\ considerations regarding a continuous rapprochement \\ RelaŢiile româno-elene în perioada interbelică. Câteva consideraŢii privind o \\ apropiere continuă
}

Radu Tudorancea

\begin{abstract}
Du moment où ont été établies des relations diplomatiques au niveau d'une légation (1879-1880) jusqu'au début de la Première Guerre mondiale, les relations roumano-grecques ont suivi une trajectoire sinueuse, marquée par quelques incidents certains d'une gravité non négligeable -, qui ont conduit à plusieurs interruptions des relations diplomatiques bilatérales. À l'évidence, le rapprochement roumano-grec devait prendre de la consistance et évoluer au fil des décennies suivantes; et cela se fit malgré des moments de tension qui ont conduit jusqu'à une interruption temporaire des relations diplomatiques dans la période 1892-1896 (dans le contexte d'un différend lié à la succession Zappa) ainsi qu'en 1906-1911 (sur fond de tensions générées par les actions de groupes armés grecs en Macédoine contre des communautés aroumaines). Les relations bilatérales sont renouées le 5 avril 1911, alors que le gouvernement roumain désignait Alexandru Florescu comme envoyé extraordinaire et ministre plénipotentiaire auprès du roi Georges à Athènes, tandis que la partie grecque nommait D. Carusso représentant diplomatique à Bucarest. Elles ont pris de la consistance, et le rapprochement est devenu si évident que les cercles occidentaux véhiculaient déjà l'idée d'un mariage dynastique entre le prince héritier Georges de Grèce et la princesse Élisabeth de Roumanie ${ }^{1}$.
\end{abstract}


2 Cependant, le rapprochement entre les deux États n'a pas conduit à la conclusion d'une alliance au moment de la Première Guerre balkanique, comme le souhaitait la Grèce, car les propositions d'Athènes au sujet d'une telle alliance ou d'une implication de la Roumanie dans la guerre ont été considérées avec réserve par le gouvernement de Bucarest. Il faut mentionner qu'au moment des guerres balkaniques, les tensions roumano-grecques étaient réapparues sur fond d'actions et d'excès commis par des groupes armés et parfois même par des éléments issus des troupes grecques contre les communautés aroumaines de Macédoine et d'Épire. La Roumanie et la Grèce devaient tout de même lutter ensemble pendant la Seconde Guerre balkanique, aux côtés de la Serbie et du Monténégro et contre la Bulgarie. De manière suggestive, le rapprochement entre Bucarest et Athènes allait être le plus évident à la fin du conflit, avec la paix de Bucarest (10 août 1913) ; en effet, la Roumanie a soutenu alors la Grèce dans ses revendications territoriales sur Kavala, tandis que le gouvernement grec assumait, pour la première fois, son engagement de respecter les droits des communautés aroumaines ${ }^{2}$.

3 En ce qui concerne la participation des deux États à la première conflagration mondiale, on peut repérer une similitude évidente. Ainsi, malgré l'influence dont jouissait à Athènes la politique de Guillaume II par l'intermédiaire de son beau-frère, le roi Constantin de Grèce, le pays a opté pour le camp de l'Entente après une période de neutralité ; cette situation est similaire à celle de la Roumanie qui avait également choisi l'Entente après une période de neutralité, malgré l'origine allemande (et implicitement l'orientation allemande) de la maison royale.

4 À la fin de la Première Guerre mondiale, les perspectives favorables pour la Grèce, consignées dans le traité de Sèvres (10 août 1920), allaient subir des transformations significatives, conséquences de la « Grande Catastrophe » d'Asie Mineure qui a conduit au traité de Lausanne (24 juillet 1923) et dont les échos furent suivis avec attention à Bucarest.

\section{Du traité de Paris à l'Entente balkanique}

5 La période de l'entre-deux-guerres peut être analysée, du point de vue des relations roumano-grecques, au fil de plusieurs étapes, en prenant bien sûr comme point de départ la Conférence de la paix de Paris des années 1919-1920. Conséquence de la solidarité des positions des deux pays ainsi que du rapprochement entre Take Ionescu et Elefthérios Venizélos, les bases étaient posées pour le projet de Petite Entente «à cinq" (Pologne, Roumanie, Tchécoslovaquie, Serbie et Grèce) qui visait à maintenir sous contrôle le péril allemand ${ }^{3}$. En vue de mettre en œuvre ce projet, le ministre roumain des Affaires étrangères a maintenu et intensifié les contacts avec les personnalités qui représentaient ces pays, avec Edvard Beneš, Nikola Pašić et, en particulier, Elefthérios Venizélos. L'amitié entre ces deux hommes politiques d'envergure était d'ailleurs de notoriété publique, et leur dialogue fut grandement facilité par leurs affinités personnelles ${ }^{4}$. Dans le passé, Take Ionescu avait affirmé à propos de Venizélos: "[...] l'ancien insurgé crétois se comportait comme un lord anglais... C'est un vrai gentleman dans toute l'acception $\mathrm{du} \mathrm{mot}^{5} »$ [en français dans le texte].

6 En ce qui concerne le projet proprement dit, Take Ionescu préconisait que soit conclu entre les cinq États un traité d'alliance garantissant l'intégrité des frontières, 
l'organisation de réunions périodiques au niveau des ministres des Affaires étrangères entre les États membres et, last but not least, une formule de coopération militaire bien organisée ${ }^{6}$. Mais rapidement, plusieurs obstacles devaient gêner son projet. D'un côté, la Pologne se trouvait engagée dans le conflit avec l'Union soviétique, tout en ayant un différend territorial grave (au sujet de la ville de Teschen) avec un autre État qui devait faire partie de l'alliance, la Tchécoslovaquie. D'autre part, la Grèce était elle aussi engagée dans la campagne d'Asie Mineure et devait affronter de multiples problèmes d'ordre politique, économique et militaire.

7 La vision de Venizélos sur les étapes à parcourir en vue du resserrement des liens entre les États de la zone balkanique avait été exposée à Take Ionescu immédiatement après la signature par la Grèce du traité de Sèvres ${ }^{7}$ (10 août 1920). Le grand homme politique grec insistait sur l'importance de l'amitié et de la solidarité entre les États de la région et montrait qu'en Grèce :

Le sentiment de solidarité entre la Roumanie, la Serbie et la Grèce pour le maintien du statu quo balkanique [...] était si profond, et l'importance d'une Roumanie forte, si conforme aux intérêts de la Grèce, que l'on ne pourrait rester impassible devant une éventuelle attaque de la part de la Bulgarie contre la Roumanie, dans le cas où les Soviétiques lanceraient une offensive contre la dernière puissance ${ }^{8}$.

8 Venizélos exprimait d'ailleurs, dans le même contexte, sa conviction que, à son tour, la Roumanie ne pourrait rester indifférente, en cas d'attaque de la Grèce par la Bulgarie'. L'homme politique grec considérait que le moment était venu d'entamer des négociations en vue de conclure une alliance défensive entre la Roumanie, la Grèce et la Yougoslavie, annonçant que dès que les élections en Grèce seraient terminées, il ferait une visite aussi bien à Belgrade qu'à Bucarest pour discuter les détails d'une telle alliance défensive ${ }^{10}$. Il est important de mentionner que, au-delà de la disponibilité manifeste de la Grèce à s'associer au projet, l'État grec était préoccupé par la proche confrontation avec les troupes kémalistes et par le fait que l'Italie avait dénoncé l'accord secret italo-grec, ce qui inquiétait la Grèce dans la perspective du conflit armé avec les troupes turques.

9 Malheureusement, des obstacles sont rapidement apparus sur la voie de ce projet régional, le plus évident étant lié au conflit territorial entre la Pologne et la Tchécoslovaquie. Quant à la participation de la Grèce, de nouvelles négociations auraient dû être organisées juste après les élections parlementaires du 14 novembre 1920, élections qui n'annonçaient aucunement le choc qu'allaient subir les libéraux de Venizélos et, implicitement, le grand homme politique grec. Malgré cette situation, les discussions concernant une éventuelle participation de la Grèce à la Petite Entente devaient se poursuivre et on peut relever à ce propos la visite à Bucarest, en juin 1923, du ministre grec des Affaires étrangères, Apostolos Alexandris.

Dans l'ensemble des relations roumano-grecques de la période de l'entre-deux-guerres, une dimension importante méritant d'être rappelée est celle des liens entre les deux maisons royales; en effet, le prince héritier de Grèce, Georges (le futur Georges II) épouse la princesse Élisabeth de Roumanie et le prince héritier de Roumanie, Carol (le futur Carol II), la princesse Hélène de Grèce; le premier mariage a été célébré à Bucarest le 27 février 1921, le second, à Athènes, quelques jours plus tard, le 10 mars 1921. Le lien entre les deux maisons royales, concrétisé par ce double mariage roumano-grec, a semblé au début devoir influencer de manière significative et positive les relations bilatérales, mais finalement, en raison de l'abandon du régime 
monarchique en Grèce ainsi que de l'apparition de conflits personnels au sein des deux couples dynastiques (tous deux ont divorcée ${ }^{11}$ ), ce ne fut pas le cas.

11 Sur le plan des relations bilatérales, après les pertes territoriales consécutives au désastre militaire de la guerre contre la Turquie de Mustafa Kemal Atatürk, la Grèce a tenté de dépasser les effets néfastes du dénouement du conflit en Asie Mineure, en essayant de se rapprocher de la Roumanie. Mais Bucarest a évité, pendant un bon moment, d'assumer des engagements vis-à-vis d'Athènes après le retour à la tête du pays du roi Constantin, l'adversaire de Venizélos. Par la suite, le rapprochement roumano-grec devait coïncider avec les négociations de Lausanne et les années suivantes qui ont été un moment d'isolement international relatif pour la Grèce après la campagne désastreuse d'Asie Mineure. L'isolement international de la Grèce s'était aggravé, dans un contexte où l'Europe avait été scandalisée par l'épisode du procès et de l'exécution, en novembre 1922, de six anciens hauts dignitaires grecs, dont deux anciens Premiers ministres, considérés comme responsables du désastre d'Asie Mineure et accusés de haute trahison.

Plus tard, l'adoption du régime républicain par la Grèce fut le motif évident d'une certaine prise de distance et d'un relâchement des liens gréco-roumains, étant donné les relations dynastiques existantes entre les deux États et la forme de gouvernement en Roumanie. Mais cette évolution fut interrompue, à l'automne 1924, par l'arrivée à Athènes $d u$ nouveau représentant diplomatique de la Roumanie, Constantin Langa-Răşcanu, un diplomate dont la longue expérience de la région balkanique a beaucoup compté pour le rapprochement et le développement des relations bilatérales entre la Grèce et la Roumanie. Il est important de mentionner le rôle particulier de ce diplomate roumain qui, au cours de sa carrière, a occupé pas moins de douze ans (1924-1936) la position d'envoyé extraordinaire et de ministre plénipotentiaire de la Roumanie à Athènes; et son activité a été remarquée aussi bien par les officiels roumains que, surtout, par les représentants de l'État grec (aussi bien durant la République qu'après le retour au régime monarchique).

L'accréditation $\mathrm{du}$ représentant diplomatique de la Roumanie à Athènes, Langa-Răşcanu, a d'ailleurs coïncidé avec une période d'aggravation des tensions gréco-bulgares. Le conflit entre la Grèce et la Bulgarie s'inscrivait dans la suite des disputes fréquentes autour du problème des minorités, qui ont impliqué l'intervention de la Société des Nations. Par le protocole Kalfov-Politis, signé par les représentants des deux États lors de l'Assemblée de la Société des Nations du 29 septembre 1924, le gouvernement d'Athènes reconnaissait l'existence d'une minorité bulgare sur son territoire. Par la suite, sous la pression de Belgrade, mais aussi de l'opinion publique grecque, Athènes a rejeté la ratification et motivé sa décision auprès de la Société des Nations par le fait qu'un tel acte aurait encouragé une action similaire en Macédoine, ainsi que par le fait que l'application du protocole aurait provoqué des frictions entre la Grèce et d'autres États. Après le rejet de ce protocole par le Parlement grec, le 3 février 1925, le Conseil de la Société des Nations en a décidé l'annulation, l'été de la même année. La Roumanie s'était intéressée de son côté aux discussions liées au protocole Kalfov-Politis, considérant qu'il pouvait être une occasion pour améliorer la situation des communautés aroumaines de Grèce.

Une autre facette de la consolidation du rapprochement roumano-grec est liée aux efforts de médiation diplomatique roumaine au sujet du conflit gréco-yougoslave des années 1924-1929. La diplomatie roumaine s'est engagée avec succès dans la médiation 
de ce conflit centré sur des questions concrètes comme celles de la zone libre de Thessalonique ou de la réglementation du trafic sur la ligne Gevgelija-Thessalonique. La participation de diplomates roumains expérimentés comme Ion Mitilineu et Langa-Răşcanu (à Belgrade et à Athènes) a contribué de manière significative à la solution du problème, fait reconnu chaleureusement par le gouvernement d'Athènes ${ }^{12}$.

Tout au long de l'entre-deux-guerres, des dissensions entre la Grèce et la Roumanie ont continué d'apparaître et se sont perpétuées au sujet de la politique des deux États à l'égard de leurs minorités nationales, le traitement des Aroumains par l'État grec constituant un sujet de tensions permanent. Il faut mentionner ici les frictions apparues après le retour des réfugiés grecs d'Asie Mineure, certaines communautés aroumaines de Grèce ayant été affectées par les mesures prises par les autorités grecques pour intégrer ces centaines de milliers de nouveaux venus sur le territoire grec.

\section{L'Entente balkanique}

16 L'année 1927 marqua pour la Roumanie la venue de Nicolae Titulescu à la tête du Ministère des Affaires étrangères, et l'intervalle entre juillet 1927 et juillet 1928, période pendant laquelle Titulescu fut responsable de ce ministère, mena à des évolutions nouvelles dans les relations roumano-hellènes. En même temps, l'appui à la cause roumaine offert par la Grèce à la Cour permanente de Justice internationale de La Haye, par l'intermédiaire du juriste grec réputé, Nikolaos Politis, fut un nouveau repère positif dans l'ensemble des relations roumano-grecques de cette période ${ }^{13}$. À leur tour, les officiels grecs donnèrent une place tout à fait privilégiée aux relations avec la Roumanie, comme le montre l'interview accordée par Andreas Michalakopoulos (titulaire du ministère des Affaires étrangères grec) au périodique Kathimerini en décembre 1927. Intitulée «La politique balkanique de la Grèce », l'interview montrait que, tandis que les relations de la Grèce avec la Turquie étaient "amicales", les relations avec la Roumanie étaient «très cordiales ${ }^{14}$ ». De plus, le ministre des Affaires étrangères grec souscrivait à l'idée lancée par Ion Gheorge Duca, qui avait déclaré que les relations entre la Grèce et la Roumanie étaient même plus cordiales que celles qui auraient pu découler d'une alliance entre les deux États ${ }^{15}$.

Progressivement, les relations roumano-grecques ont repris un cours ascendant qui devait se concrétiser par la signature du Pacte de non-agression et d'arbitrage entre la Grèce et la Roumanie le 21 mars 1928. Les parties contractantes s'engageaient réciproquement à ne pas s'attaquer, à ne pas envahir le territoire de l'autre, à ne pas se déclarer la guerre sauf en cas de légitime défense, dans le cas d'une action pour appliquer l'article 16 du pacte de la Société des Nations ou dans le cas d'une action nécessitée par une décision prise par l'Assemblée ou le Conseil de la Société des Nations. Nicolae Titulescu avait soutenu la conclusion d'un traité d'arbitrage avec un autre État (en l'occurrence avec la Grèce) comprenant des clauses plus «étroites » que celles du traité signé avec la France; en effet, pensait-il, si l'on demandait à l'avenir à la Roumanie de signer un traité sur la base de celui passé avec la France, Bucarest pourrait prendre pour modèle le traité roumano-grec et répliquer que, dans le cas du traité signé avec la France, le document avait des clauses larges parce qu'il était le «complément d'un traité d'alliance ${ }^{16}$ ». À son tour, Andreas Michalakopoulos, revenu de Genève au début du mois d'avril, fit des 
déclarations à la presse grecque et étrangère au sujet de la signature du Pacte de non-agression et d'arbitrage entre la Roumanie et la Grèce, et de ses significations. Selon le ministre grec des Affaires étrangères, le pacte montrait que la politique extérieure hellène, "après l'abolition de la dictature ", avait commencé à porter ses fruits $^{17}$. Michalakopoulos notait aussi que bien qu'entre la Roumanie et la Grèce il n'y eût pas de "problèmes sérieux", ceci n'empêchait pas qu'Athènes apprécie la "signification morale» du pacte qui venait d'être conclu ${ }^{18}$.

Incontestablement, le moment de la signature du pacte constitue une étape importante des relations gréco-roumaines de cette période, d'autant plus qu'il s'agissait du premier pacte de ce type signé par deux États de la région. La signature du document de mars 1928 ouvrait la voie à d'autres accords interbalkaniques. Ceux-ci devaient conduire au rapprochement des États de la région, qui s'est d'ailleurs concrétisé par les Conférences balkaniques et, finalement, par la signature à Athènes du pacte de l'Entente balkanique le 9 février 1934.

19 L'apogée des relations gréco-roumaines est représenté par la visite en Roumanie du Premier ministre grec, personnalité grecque la plus influente de l'entre-deux-guerres (qui avait déjà influencé l'évolution de la Grèce avant la Première Guerre), Elefthérios Venizélos, en août 1931, à l'invitation du Premier ministre roumain de cette période, Nicolae Iorga, qui remplissait en même temps la fonction de président de l'Association roumaine des amis de la Grèce. Au-delà de l'enthousiasme général ressenti tant à Bucarest qu'à Athènes à l'occasion de cette visite, du côté des résultats concrets il faut inscrire la signature de la Convention sur le commerce et la navigation entre la Roumanie et la Grèce et, en particulier, l'octroi de la clause de la nation la plus favorisée, de manière illimitée et inconditionnelle.

Non moins importante (si l'on prend en compte l'accueil impressionnant et les réactions positives dans la société grecque) fut la visite du ministre des Affaires étrangères roumain, Nicolae Titulescu, à Athènes à l'automne $1933^{19}$. Menée dans le contexte des efforts pour concrétiser le rapprochement entre la Roumanie, la Grèce, la Yougoslavie et la Turquie, la visite du ministre roumain a déclenché un enthousiasme remarquable dans la population athénienne, Titulescu étant ovationné par des dizaines de milliers d'habitants tout au long de la rue Stadiou, l'une des principales de la capitale grecque.

21 Un chapitre important des relations roumano-grecques est constitué par les Conférences balkaniques (la première Conférence a eu lieu à Athènes du $5 \mathrm{au}$ 12 octobre 1930), réunions au caractère non gouvernemental qui ont préfiguré la naissance de l'Entente balkanique. La signature, le 9 février 1934, du pacte de l'Entente balkanique, par les ministres des Affaires étrangères de la Roumanie, de la Turquie, de la Yougoslavie et de la Grèce à Athènes devait conduire à un rapprochement plus fort encore entre la Roumanie et la Grèce, cette fois sous la tutelle de cette structure. Il faut souligner que la participation de la Grèce comme celle de la Roumanie à l'Entente balkanique reposaient sur l'intérêt commun des deux États à maintenir le statu quo territorial et à contrebalancer le courant révisionniste de plus en plus fort dans l'Europe de cette période. Pour revenir aux stipulations du pacte, on sait que l'article $3 \mathrm{du}$ protocole annexe (article qui mentionnait la situation dans laquelle, si l'une des parties contractantes était victime d'une agression de la part d'un État non balkanique, le pacte devenait effectif) fut à l'origine de fortes tensions entre le ministre grec des Affaires étrangères, Dimitrios Maximos, et l'opposition grecque (en particulier 
Venizélos), ce qui causa de l'inquiétude du côté roumain, entre autres. Les craintes de Venizélos avaient pour cause une hypothétique attaque de l'Italie contre l'un des signataires, éventualité dans laquelle la Grèce, selon le pacte, aurait été obligée d'intervenir, ce qui la rendait extrêmement vulnérable étant donnée sa position géographique. Malgré les hésitations de la Grèce vis-à-vis de l'Entente balkanique, dues à l'influence puissante de Venizélos sur la politique du gouvernement d'Athènes, c'est justement la Grèce qui allait profiter la première du soutien des autres membres de l'Entente dans les moments critiques qui suivirent la tentative de «révolution républicaine " de Venizélos, quand le courant revanchard bulgare, profitant de la situation ambiguë de la Grèce, devint extrêmement véhément et menaçant. Très rapidement, Nicolae Titulescu (président en exercice de l'Assemblée générale de la Société des Nations, à ce moment-là) devait faire savoir à la Bulgarie que dans l'éventualité où les forces bulgares pénétreraient en Grèce, «le Pacte balkanique entrerait automatiquement en application ${ }^{20} »$.

\section{Liens économiques, échanges commerciaux}

D'autre part, dans l'ensemble des relations roumano-grecques, les liens économiques entre les deux pays eurent un rôle important, de sorte que l'évolution des échanges commerciaux, leur profil et leur dynamique furent autant d'éléments qui complètent cette image des relations bilatérales. Une caractéristique fondamentale de ces relations économiques entre la Grèce et la Roumanie pendant l'entre-deux-guerres est le fait que la balance commerciale a continué d'être massivement déficitaire pour la Grèce, malgré les efforts faits lors des sessions du conseil économique de l'Entente balkanique. Le volume des échanges entre les deux pays a évolué au cours de la période, mais le plus haut niveau fut enregistré en 1928. Dans l'intervalle 1928-1929, la Roumanie fut le plus grand exportateur sur le marché grec de maïs, mais aussi de bois de construction, de pétrole et de produits pétroliers, domaine où elle détenait un quasi-monopole. Si l'on parle en termes de hiérarchie, la Grèce occupait la $6^{\mathrm{e}}$ place sur les 26 pays importateurs de produits roumains. Incontestablement, le moment le plus important fut la signature, en août 1931 à Bucarest, de la Convention de commerce et de navigation ${ }^{21}$ entre les deux pays. Mais ses effets positifs ont été atténués par la crise économique mondiale des années 1929-1933. Bien que celle-ci ait affecté les deux pays, la Grèce fut cependant plus touchée pour deux raisons: d'une part, à cause du profil des exportations grecques, concernant des produits considérés comme « de luxe » (huile d'olive, raisins secs, tabac, condiments) pour lesquels la demande internationale a baissé à cette période, d'autre part parce que la Grèce avait en permanence une balance commerciale déficitaire. La position de la Roumanie sur le marché grec a résisté grâce à la structure générale de ses exportations représentées par des produits de première nécessité, dont l'État grec ne pouvait se dispenser.

Sur le plan économique, on peut observer également une évolution relativement proche de la situation des deux pays par rapport à l'offensive économique allemande. Dans ce domaine, la Roumanie et la Grèce ont dû accepter la consolidation de la position allemande dans leurs échanges commerciaux à la suite d'accords dont les clauses étaient comparables.

En ce qui concerne l'Entente balkanique ${ }^{22}$, dans laquelle les deux pays se sont engagés au cours des années 1930, celle-ci devait montrer ses limites et échouer à l'occasion des 
moments critiques vécus par les pays membres, dénouement favorisé par un ensemble de facteurs régionaux et internationaux. On peut dire que cet échec a été causé par l'apparition de situations (auxquelles les pays membres se sont heurtés) différentes de celles qu'avaient anticipées les initiateurs du pacte au moment où furent fixés les accords politiques et les dispositions militaires.

$\mathrm{Au}$ contexte international et aux positions adoptées par les grandes puissances allait s'ajouter une série de "défaillances » dans la manière d'aborder la politique étrangère parmi les membres de l'Entente. L'exemple classique est celui de la Yougoslavie du gouvernement Stojadinović signant le 24 janvier 1937 un pacte d'« amitié permanente et sincère » avec la Bulgarie sans solliciter le consentement de ses alliés comme il était prévu dans le pacte.

\section{Conclusions}

S'il faut évaluer la période de l'entre-deux-guerres dans son entier, on peut affirmer que Bucarest comme Athènes ont eu des objectifs similaires en matière de politique étrangère, leur principal objectif étant le maintien du statu quo territorial. Alors que pour la Roumanie, la menace était perçue comme venant du développement des tendances révisionnistes en Bulgarie et en Hongrie, dans des conditions où, à l'Est, la menace soviétique persistait, dans le cas de la Grèce, au-delà des prétentions de la Bulgarie, la menace la plus forte venait de l'Italie de Mussolini. La peur de provoquer ou d'attirer des suspicions à Rome fut précisément un leitmotiv de la politique étrangère grecque avant même la signature du pacte de l'Entente balkanique. D'où les problèmes apparus au moment de la ratification du Pacte balkanique ainsi que la pression exercée par l'opposition grecque (Venizélos en tête) qui a conduit à produire une « déclaration interprétative ${ }^{23}$ » du pacte dont le rôle était d'écarter la variante selon laquelle à un moment donné la Grèce serait contrainte de s'engager dans un conflit avec l'Italie. Les mêmes craintes ont empêché initialement la Grèce de participer à la constitution d'une Convention militaire à quatre dans le cadre de l'Entente balkanique; lorsqu'elle a décidé de participer tout de même, la Grèce l'a fait en tenant compte des conditions (limitatives) exposées par Ioannis Metaxás dans sa lettre de mai 1936.

Une différence significative entre les deux États du point de vue de leurs réactions dans des moments critiques devait apparaître au moment où Roumanie et Grèce ont dû faire face aux ultimatums soviétique et italien de l'année 1940. Alors que la Roumanie a cédé sans lutte des territoires substantiels (il est vrai, pour sauvegarder l'État), la Grèce a choisi, et sans même tenir compte du délai de trois heures accordé par l'Italie au moment de l'ultimatum (le 28 octobre 1940), de le rejeter et de riposter contre l'envahisseur.

28 Au moment de l'agression italienne contre la Grèce, la Roumanie avait déjà modifié le cours de sa politique étrangère (dans des conditions où, après les pertes territoriales de l'été 1940, ses nouvelles frontières étaient garanties par l'Allemagne et l'Italie) et les vieux engagements qui la liaient à la Grèce n'existaient plus. D'ailleurs, l'occupation de la Grèce par les forces allemandes allait déterminer, le 24 juin 1940, la rupture des relations diplomatiques entre la Roumanie et la Grèce, mais il faut rappeler que la représentation de la Roumanie à Athènes a continué de fonctionner jusqu'au 15 juin 1944, date à laquelle elle fut transformée en Consulat général. 
Évaluant l'ensemble de la période considérée, on peut malgré tout affirmer qu'à partir de l'automne 1924, les relations bilatérales roumano-grecques ont suivi clairement une tendance ascendante, en dépit des problèmes bilatéraux d'ordre économique et de questions liées au traitement des populations aroumaines de Grèce. Cependant, la question des droits des Aroumains et leur traitement par les autorités grecques sont restés à l'ordre du jour des relations bilatérales durant toute la période, même si ce fut dans une proportion plus faible.

Comme on peut le voir, la période de l'entre-deux-guerres s'est caractérisée, au niveau européen, par la confrontation entre le camp des pays décidés à maintenir le statu quo territorial hérité des traités de paix (le système de Versailles) et ceux qui visaient sa révision. Dans cette dispute, la Grèce comme la Roumanie se sont trouvées du même côté, avec des objectifs communs, à savoir la défense de leur souveraineté nationale et de leur intégrité territoriale. Cette solidarité d'intérêts des deux pays s'est consolidée durant toute la période et a été renforcée par le rapprochement dynastique entre les deux pays et, surtout, par les relations d'amitié privilégiées établies au niveau des élites politiques et diplomatiques des deux États.

\section{BIBLIOGRAPHIE}

L'étude est fondée principalement sur les ressources des archives des ministères français et roumain des Affaires étrangères.

On peut ajouter :

GRENVILLE John Ashley Soames, 1974, The Major International Treaties 1914-1973. A History and Guide with Texts, London: Methuen \& Co. Ltd.

IONESCU Take, 1921, Mica ÎnŢelegere, București: Cartea Românească.

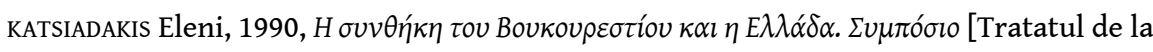

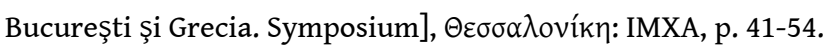

XENI Constantin, 1932, Take Ionescu, 1858-1922, Bucureşti: Editura Universul.

\section{NOTES}

1. Archives du Ministère des Affaires étrangères de Roumanie (Arhiva Ministerului Afacerilor Externe al României), Fonds 71/R/100, f. 106.

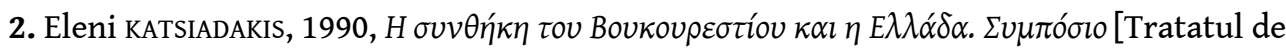

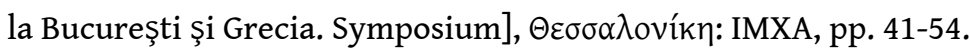

3. Constantin XENI, 1932, Take Ionescu, 1858-1922, Bucureşti: Editura Universul, p. 465.

4. D'après le témoignage de Take Ionescu, les deux hommes politiques se sont rencontrés pour la première fois à Londres en janvier 1913, invités tous deux à un 
déjeuner par un célèbre journaliste anglais, Emile Joseph Dillon, vieil ami de Venizélos. On trouve des références intéressantes dans une préface d'un ouvrage de Costas Kérofilas publié à Paris (qui porte sur la personne de Venizélos), préface dont l'auteur n'est autre que Take Ionescu. Celui-ci souligne notamment: "[...] j'ai eu l'occasion de voir Venizelos avoir raison contre tout le monde et imposer sa volonté pour le plus grand bien de sa patrie... je fus dès le premier moment attiré. Cette tête de saint byzantin qu'on dirait descendue d'une fresque d'église, ce regard à la fois si pénétrant et si tendre, ce sourire si fin, cette sympathie irrésistible qui se dégage de toute sa personne, cette modestie presque de jeune fille et qui est d'autant plus charmante qu'elle est alliée à une volonté de fer, tout cela vous prend à la première rencontre. Je lui demandai le secret de son succès, et il me répondit ces paroles si simples mais si profondes : “j’ai toujours dit à mes concitoyens toute la verité, toute, et je me suis toujours senti prêt à quitter le pouvoir sans aucun regret". La sincérité, le culte de la vérité, voilà la plus belle des qualités de Venizelos, et en même temps le secret de sa force. » (Costas KÉRofilas, 1915, Un homme d'État: E. Venizélos, sa vie, son œuvre, Paris : Éditions de l'Imprimerie de l'Est, p. 7).

5. Ibid., p. 8. Take Ionescu évoque à cette occasion l'échange de télégrammes entre eux des 13 et 15 juin 1913, soulignant, dans la préface citée, que ceux-ci « ont posé les bases de toute la politique gréco-roumaine de l'année 1913 ».

6. Take IONESCU, 1921, Mica ÎnŢelegere, București: Cartea Românească, pp. 6-7.

7. „Prevederile Tratatului de Pace dintre Puterile Aliate şi Asociate şi Turcia (Tratatul de la Sèvres)-10 august 1920", in John Ashley Soames GRENVILLE, 1974, The Major International Treaties 1914-1973. A History and Guide with Texts, London: Methuen \& Co. Ltd, pp. 73-77.

8. Archives de la Bibliothèque nationale (Roumanie), Fonds Saint-Georges, P. VIII, D 7, f. 49,2 sept. 1920.

9. Ibid.

10. Ibid. Dans le texte : «[...] le temps est peut-être venu d'entamer des négociations en vue de la conclusion rapide d'une alliance défensive entre la Roumanie, la Yougoslavie et la Grèce, visant une éventuelle attaque de la part de la Bulgarie. En vérité, une telle alliance empêcherait la Bulgarie de se lancer dans une politique aventurière et sera, en Grèce du moins, unanimement saluée ; elle supprimerait en même temps tout prétexte de contradiction de la part de l'opposition qui pourrait chercher à tirer un profit politique en s'opposant à l'entrée en guerre de la Grèce aux côtés de la Roumanie si l'obligation contractuelle d'une alliance n'existait pas entre les deux pays. Étant absorbé par le traité avec la Turquie et sans penser que le problème soit urgent, j'ai l'intention de me rendre à Bucarest et à Belgrade, vers la fin du mois d'octobre ou le début du mois de novembre, une fois terminées les élections en Grèce. »

11. Pour les premiers signes de l'échec, en ce qui concerne les mariages dynastiques, voir Archives du Ministère des Affaires étrangères (AMAE), Fonds Romania, 71-1922, R 50 - dossiers spéciaux, f. 19, Rapport $\mathrm{n}^{\circ}$ 507-5, 31 janvier 1923, Légation de la Roumanie aux États-Unis, Washington. Le divorce entre le prince Carol de Roumanie et la princesse Hélène de Grèce fut prononcé en juin 1928, celui de George de Grèce et Élisabeth de Roumanie en 1935.

12. AMAE, Fonds Grecia 71/1925, Vol. 117/2, G 6 A, Rapport n 2043, 11 décembre 1926, Légation roumaine d'Athènes, signé par Langa Rascanu, f. 48. 
13. AMAE, Fonds Conv., D 3, Vol. 4, Lettre de Nicolae Titulescu du 3 décembre 1927 à Nikolaos Politis, envoyé extraordinaire et ministre plénipotentiaire de la Grèce à Paris, par laquelle le ministre des Affaires étrangères roumain le remerciait pour l'appui offert à la Roumanie à la Cour permanente de Justice internationale de La Haye.

14. AMAE, Fonds Grèce, Vol. I, Rapport $n^{\circ} 2554$ du 28 décembre 1927 de la Légation de la Roumanie à Athènes, signé par Langa Răşcanu, contenant en annexe l'interview accordée par Andreas Michalakopoulos au journal Kathimerini, au sujet de la politique extérieure de la Grèce, f. 187.

15. Ibid.

16. AMAE, Fonds 9, Conventions G. 3, Télégramme du 14 mars 1928 de Nicolae Titulescu, ministre des Affaires étrangères, à I. G. Duca, au sujet du Pacte de non-agression et d'arbitrage roumano-grec.

17. AMAE, Fonds $71 /$ Grèce, Vol. I, Rapport $n^{\circ} 974$ de la Légation de la Roumanie à Athènes du 9 avril 1928, contenant aussi une annexe signée par Langa Răşcanu, f. 218-219.

18. Ibid.

19. AMAE, Fonds $71 /$ Grèce, Vol. III, Rapport $n^{\circ} 2908,28$ novembre 1933, Légation roumaine d'Athènes, signé par Langa Răşcanu.

20. AMAE, Fonds $71 /$ Grèce, Vol. V, Télégramme du 10 mars 1935, signé par Nicolae Titulescu.

21. AMAE, Fonds Atena, Vol. 76 bis, Convenţiunea de ComerŢ şi de NavigaŢie dintre România şi Grecia, 11 août 1931, p. 403.

22. Sur la position de la Grèce vis-à-vis de l'Entente balkanique, voir AMAE, Fonds ÎnŢelegerea Balcanică, Dos. 14, Rapport nº 623, 8 mars 1934, f. 235-236.

23. AMAE, Vol. 19, Télégramme $n^{\circ}$ 695, 6 mars 1934, Légation de la Roumanie, Athènes, signé par Constantin Langa Răşcanu.

\section{RÉSUMÉS}

Avec l'apaisement des tensions diplomatiques de la fin du XIX ${ }^{e}$ siècle et du début du Xx $x^{e}$ siècle, les relations roumano-grecques ont suivi un cours sinueux dans l'entre-deux-guerres, influencé par les changements politiques internes et les événements européens. Deux mariages dynastiques, bien que de courte durée, entre les maisons royales des deux pays, des liens privilégiés entre certains gouvernants (Elefthérios Venizélos et Take Ionescu), l'action de représentants diplomatiques compétents (Constantin Langa-Răşcanu à Athènes), mais surtout des intérêts communs à une politique de coopération dans les Balkans, dans un contexte où la Grèce pâtit des effets du désastre d'Asie Mineure, préparent le terrain à la signature du traité de non-agression et d'arbitrage de mars 1928 et à l'organisation de conférences balkaniques ainsi que, plus tard, au pacte de l'Entente balkanique de 1934. Les visites d'Elefthérios Venizélos en Roumanie en août 1931 puis de Nicolae Titulescu (ministre roumain des Affaires étrangères) à Athènes en 1933, où l'accueil fut enthousiaste, marquent l'apogée des relations bilatérales roumano-grecques. Sur 
cette période, les relations entre les deux États montrent ainsi un cours ascendant malgré le maintien dans l'agenda bilatéral de questions sensibles comme celle des Aroumains ou certains aspects techniques des échanges économiques.

After the diplomatic tensions of the XIX ${ }^{\text {th }}$ Century, the Romanian-Greek relations have followed, during the interwar decades, a winding course, influenced either by the internal political changes in the two states or by the international events occuring in Europe. The ties between the two Royal Houses, materializing in the Romanian Greek dynastic marriages, excellent relations between outstanding political men (Eleftherios Venizelos et Take Ionescu), the arrival in Athens of Romania's new minister, Constantin Langa-Răşcanu, a diplomat with a large experience in the Balkan area and, of course, common economical interests were to help put the relations between Romania and Greece back on a favorable track. A milestone in the overall course of Romanian-Hellenic relations was that of the signing of the non-aggression and arbitrage pact, in March 1928; it paved the way for other inter-Balkan agreements leading to rapprochement between the Balkan states, which materialized in the signing of the Balkan Entente Pact on February 9, 1934. However, the peak in the Romanian-Hellenic relations were the Romanian visit of Greek Prime Minister E. Venizelos (August 1931) and the visit of Romanian Foreign Minister Nicolae Titulescu to Athens (Autumn of 1933) - a decisive moment, considering the impressive welcome he enjoyed. Overall, it can be said that, from the Autumn of 1924 and until the outbreak of the Second World War, the Romanian-Greek bilateral relations obviously followed an upward trend, in spite of some minor issues related to how the Aromanian populations were treated or some strictly technical economic problems.

Odată cu depăşirea tensiunilor diplomatice de la sfârşitul secolului al xIX-lea şi începutul secolului al xx-lea, relaţiile româno-elene în perioada interbelică au urmat un curs sinuos, influenţat de schimbările politice interne şi de evenimentele care au survenit în Europa. Legăturile dintre cele două Case regale, care s-au concretizat în cele două căsătorii dinastice (chiar dacă de scurtă durată), relaţiile privilegiate între personalităţi politice (precum Eleftherios Venizelos şi Take Ionescu), activitatea unor reprezentanţi diplomatici competenţi (Constantin Langa-Răşcanu la Atena), dar mai ales interesele comune privind o politică de cooperare în Balcani, în contextul în care Grecia resimŢea încă efectele dezastrului din Asia Mică, au pregătit terenul pentru semnarea Tratatului de neagresiune şi arbitraj din martie 1928 şi pentru organizarea conferinŢelor balcanice, ca şi pentru parafarea Pactului ÎnŢelegerii Balcanice, în februarie 1934. Vizita lui Elefthérios Venizélos în România (august 1931) și cea a lui Nicolae Titulescu (ministrul Afacerilor Externe al României) la Atena, în 1933, unde primirea a fost una entuziastă, au marcat apogeul relaţiilor bilaterale româno-elene. În perioada la care facem referire, relaŢiile dintre cele două state au urmat un curs ascendent, în ciuda menȚinerii pe agenda bilaterală a unor chestiuni sensibile, precum cea a drepturilor aromânilor, sau a unor aspecte tehnice legate de schimburile economice. 


\section{INDEX}

Index géographique : Grèce, Roumanie

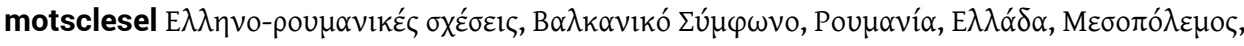
$\Delta \imath \pi \lambda \omega \mu \alpha \tau \imath \kappa \eta ́ ~ \iota \sigma \tau o \rho i ́ \alpha$

Thèmes : Histoire diplomatique

Keywords : Greek-Romanian relations, Balkan Pact, Greece, Romania, Interwar period, Diplomatic History

motsclesmk ГРЦИЈА /РОМАНИЈА ОДНОСИ, БАЛКАНСКИОТ ПАКТ, РОМАНИЈА, ГРЦИЈА, ДИПЛОМАТСКА ИСТОРИЈА, МЕЃУВОЕНИОТ ПЕРИОД

Mots-clés : relations gréco-roumaines, relations gréco-roumaines, Entente balkanique, Ionescu Take (Demetriu) (1858-1922), Venizélos Elefthérios (1864-1936)

motsclestr Yunan-Romen ilişkileri, Balkan Antantı, Romanya, Yunanistan, İki savaş arası dönem, Diplomatik Tarih

Index chronologique : entre-deux-guerres (1918-1939)

\section{AUTEUR}

\section{RADU TUDORANCEA}

Institut d'Histoire « Nicolae Iorga », Bucarest 